\title{
Peripheral immune tolerance's orchestration in human pregnancy: suggestions for tissue and organ transplantation
}

\author{
Giacomo Manfredi* \\ Department of Clinical Medicine, Religious Gen. Reg. Hospital “F. Miulli”, Acquaviva delle Fonti (BA), Italy
}

\begin{abstract}
The pregnancy is a very big challenge for the human immune system. To ensure the embryo's survival anyway in spite of its semiallogeneic heritage, the mother plays many games on several fields: these games are played under hormonal supervision and are aimed both to ensure the right embryo's nutrition and growth, and to stop the specific mother's cellular and humoral immune responses against embryo's paternal antigens. To attain these goals hormonal, metabolic, vascular, cellular and humoral immune mechanisms are put in play by the mother's body: the result is the birth of a new life. To follow in pregnancy's immune tolerance's footsteps may be very helpful in reaching long term survival of transplanted tissue and/or solid organ in humans.
\end{abstract}

\section{Peripheral immune tolerance mechanisms in human pregnancy}

\section{Histocompatibility antigens and immune tolerance}

To carry out the pregnancy happily, the woman plays many games on several fields; but all these changes are elicited by the unique biologic behavior of the fertilized egg. Really, thanks to soluble and decidual mother-derived Epidermal Growth Factor (EGF) and to its own EGF Receptors, the blastocystis plays first adhesion to, and then invasion of, the maternal decidua. After these early events, the extraordinary immunologic interaction between two leading actors begins: to the villus formation, attained thanks to Vascular Endothelial Growth Factor (VEGF), the birth of new spiral arteries into the maternal decidua follows, and the trophoblast (cytotrophoblast and syncytiotrophoblast) invades the maternal decidua by entering both intravascular and extravascular space. At this phase the trophoblast cells just express HLA isoform E, F and G [1] without showing classic class I $(A, B, C)$ HLA; the expression of HLA-G1 on the antigen presenting cells induces immunosuppressive $\mathrm{CD} 4^{+} \mathrm{T}$ cells [2], while the continuous and increasing shedding of soluble HLA-G (sHLA-G) ensures an effective and specific anergy of $\mathrm{T}$ cell mediated cytotoxicity $[3,4]$. This mechanism is the same utilized by metastatic colon cancer cells to attain immune escape by inducing $\mathrm{T}$ cell anergy and achieve vascular invasion [5-9].

\section{Sex hormones and immune tolerance}

The normal pregnancy couples the down-regulation of estrogens until to quite silencing them. Otherwise the progesteron synthesis increases more and more during pregnancy and its binding on specific receptors, shown both on CD4 and CD8 T cells, induces inhibition of $\mathrm{T}$ cell proliferation, down-regulation of IFN- $\gamma$ and TNF- $\alpha$ and up-regulation of IL-4 synthesis; these effects are directed on T cells and are not antigen-specific [10]. Moreover, progesterone-engaged lymphocytes synthesize a $34 \mathrm{k}-\mathrm{Da}$ protein named ProgesteronInduced Blocking Factor (PIBF), able to inhibit arachidonic acid and NK activity; PIBF induces also nuclear translocation of STAT6 and down-regulates the STAT-4 phosphorylation, so inducing the switch from Th-1 to Th-2 cytokine pattern secretion [11]. On the other hand, testosterone has shown immunosuppressive effects, by downregulation of both innate and adaptive cell-mediated immunity [12]. Estrogens showed up-regulation in heparanase genes expression during breast cancer resulting in a prothrombotic state [13]; coagulation's activation is related to immune phlogosys and act as an important mechanism involved in immune rejection. The sex hormones-induced modulation of immune response gives the reason for sex differences in the prevalence and clinical outcome of autoimmune diseases.

\section{Metabolic mechanisms}

The right embryo's growth is guaranteed by the wide and full supplementation of nutritional factors. Both glucidic, proteic and lipidic support, together with all factors enhancing DNA synthesis, are indispensable to ensure the right cells growth. It's well established that the non-supplementation of folic acid and vitamin B12 during the pregnancy may bring forward abnormal cell proliferation and differentiation with possible defect in the neural tube shutting [14]. Really the folic acid also showed a pivotal role in the Nitric Oxide bioavailability thanks to its active metabolite 5-methyl tetrahydrofolate: the folic acid-driven enhancement of endothelial NO synthase and NO production acts as superoxide radicals scavenger and improves endothelial functions, so ensuring optimal tissue's perfusion [15]. Moreover, in pre-eclamptic pregnancies Vitamin D reduces placental trophoblasts oxidative stress and increases endothelial Nitric Oxide synthesis [16].

${ }^{*}$ Correspondence to: Manfredi G, Department of Clinical Medicine, Religious Gen. Reg. Hospital "F. Miulli", Acquaviva delle Fonti (BA), Italy, Tel: 0039-805277317; E-mail: giacomo.manfredi@alice.it

Key words: peripheral immune tolerance, sex hormones driven immune modulation, gut microbioma role in immune tolerance, immunoglobulins and idiotype network's role in immune tolerance

Received: June 07, 2018; Accepted: June 19, 2018; Published: June 22, 2018 


\section{Vascular mechanisms}

Both VEGF-driven angiogenesis and $\mathrm{NO}$ as well as $\mathrm{H} 2 \mathrm{~S}$ production by uterine arterial endothelial cells are improved in pregnant as compared to non-pregnant woman [17]. These effects show the relationship between vasodilation and angiogenesis in the protection and right embryo's growth. Endothelial dysfunction in pre-eclamptic pregnant women has been related to impaired production and blood levels of VEGF [18]; the main VEGF role in pregnancy is the angiogenesis stimulation and the right coagulation control. During pregnancy, the complement activation via alternative pathway involves coagulation activation, so that the right endothelial function including plasminogen activation and fibrinolysis is essential to prevent thrombosis and ensure the right blood flow [19]. The endothelial dysfunction leads both to activation of coagulation as showed by blood d-dimer increase and to impairment of NO synthase and production, as happens in pre-eclampsia [20].

Of course the coexistence of a thrombophylic status (as happens in hyperomocysteinemia, Hughes Syndrome and all genetic driven coagulation diseases) may jeopardize the right embryo's blood flow, so endangering the new life survival [21-25].

\section{Cell-mediated mechanisms}

Regulatory T cells (Treg) play a pivotal role in the achievement and keeping of peripheral immune tolerance. During pregnancy, it has been shown that Treg functions are related to the expression of an inhibitory costimulatory molecule named PDL-1; thanks to the expression of PDL1 on Treg, there is an inhibition in the synthesis of IFN- $\gamma$ and IL-17, as well as an increase of FOXP3+CD4+CD25+ [26], the Treg phenotype acting by synthesis of the tolerogenic molecule IL-10. Moreover, Treg are chemoattracted to the site of contact between paternal antigens and maternal immune cells by Human Chorion Gonadotropin (HCG) secreted by blastocyst [27]; the selective migration of Treg into the uterus establishes the destiny of the following tolerogenic mathernal immune response. On the other hand, uterine NK cells produce angiogenic factors such as VEGF, placental growth factor (PlGF) and the tolerogenic cytokine TGF- $\beta$ and can play a role in the induction of paternal MHC class I tolerance; during normal pregnancy, T cells reacting with autoantigens are removed by Fas ligand/Fas-mediated apoptosis, a process known as 'clonal deletion', leading to tolerance. HLA-G and the Fas ligand expressed on trophoblasts may play a role in clonal deletion: soluble HLA-G1 produced by syncytiotrophoblasts can achieve immune tolerance to fetal or paternal antigens by inducing apoptosis of activated maternal CD8+ T cells via the Fas ligand/ Fas system. Lack of or decrease of soluble HLA-G1 expression in trophoblasts may cause inadequate tolerance at the level of fetomaternal interface resulting in pre-eclampsia [28].

\section{Humoral mechanisms}

Prolonged exposure of the female reproductive system to seminal fluid with certain immunogenetic features may alter the immune response and decrease the risk of pre-eclampsia in the case of pregnancy by that immunogenetic features. Vaginal or uterine antigen presenting cells (APC) may take up soluble major histocompatibility complex (MHC) class I antigens present in the seminal fluid leading to specific tolerance to paternal MHC class I antigens [28]. Moreover, between humoral factors influencing a right immune tolerance in pregnancy, there is the idiotype/antiidiotype network balance: in women suffering from recurrent pregnancy loss, after excluding any other causes as coagulation disorders or autoimmunity diseases, the imbalance of IgG subclasses, mainly IgG1 and IgG3, was the leading cause of pregnancy loss and the i.v. infusion of low dose whole Immunoglobulins $(200 \mathrm{mg} /$ $\mathrm{kg} / \mathrm{month}$ ) all over the pregnancy allowed to carry out the pregnancy happily in more than $90 \%$ of patients [29]. The molecular features of IgG1 and IgG3, thanks to their flexibility, make them suitable to act as anti-idiotypic antibody: the right balance between these antibodies guarantees the right modulation of functions of many biological receptors. Actually the idiotype-antiidiotype network may recognize and modulate the functions of virtually all biologic receptors; really in the above mentioned study both the bleeding and also the abnormal hypercontractility of the uterus, resistant to all tocolytic treatments, were fully bridled thanks to i.v. immunoglobulins treatment, showing that also the muscarinic receptors of the uterus are sensitive to idiotypic network's modulation. Moreover, the administration of two peptides having antiidiotype activity on an epitope (16/6) of a pathogenic antidsDNA antibody in a murine model of and in human patients suffering from Systemic Lupus Erythematosus (SLE), attained both prevention and treatment of established SLE [30] thanks to upregulated secretion of the regulatory cytokine TGF- $\beta$, so showing the importance of idiotypic network in the modulation of immune response in autoimmunity, too.

\section{Oral tolerance}

The allergen exposition to oral mucosa has been shown to induce immune tolerance thanks to the unique immune features of vestibular Antigen Presenting Cells (APC); really the prolonged exposition of antigen to these cells induces activation of regulatory $\mathrm{T}$ cells (T-reg) thanks to improved synthesis of tolerogenic cytokines as IL10 and inhibition of IL-4, IFN- $\gamma$ IL-2 and IL-13 [6]. Moreover the oral exposition of ovalbumin in sensitized mice showed tolerogenic properties inducing down-regulation of innate type 2 lymphoid cells infiltration in atopic dermatitis [7]. The oral tolerance way has been taken to treat human autoimmune diseases as multiple sclerosis, arthritis, uveitis and diabetes and in pollens and food allergy $[8,9]$. Maybe the erotic practices including deep kisses with exchange of biologic fluids as spittle may help the mother to achieve immune tolerance against paternal antigens thanks to these mechanisms.

\section{The gut microbioma's effects on immune system}

In autoimmune diseases as Experimental Autoimmune Encephalomyelitis (EAE), showing in mice the same pathology as Multiple Sclerosis in humans, the administration of probiotics as Lactobacilli shows a tolerogenic effect both on mesenteric lymphnodes and on central nervous system thanks to increase of IL-10 synthesized by Treg [21]. Also another commensal bacterium of intestinal microbiota, Bacteroides fragilis, showed tolerogenic properties inasmuch as it synthesize Polisaccharide A (PSA) that binds Toll Like Receptor 2 on T cells, so increasing the synthesis of IL-10 by enhanced Foxp3+CD4+T cells, the classical Treg phenotype; so doing, the PSA is not only able to prevent, but also to cure experimental colitis in animals [22]. Moreover the dietary supplementation of Lactobacillus Rhamnosus GG to extensively hydrolyzed cow's milk in infants suffering from cow's milk allergy, results in a higher rate of tolerance acquisition as compared to those treated with extensively hydrolyzed cow's milk alone [23]. The ligands by witch microbiota binds lymphocytes are Toll Like Receptors (TLR), and the engagement and activation of different TLR achieve different effects on immune system: the lipopolysaccharide (LPS) engages TLR4 and achieves immune response against Gram-negative bacteria, while components of Gram-positive bacteria bind TLR2. Normally the expression of TLR2 and TLR4 are confined to crypts 
of the mouse gut mucosa, while only the cells on the lumen express TLR3, interacting with commensal microbioma as B. Bifidum and B. Fragilis and its engagement makes the gut mucosa able to strengthen an antiviral response thanks to IFN- $\beta$ synthesis [24]. On the other hand the administration of antiLPS enriched colostrum, picked up from cows immunized by LPS derived from same strains of intestinal Escherichia Coli bacteria, alleviated bowel inflammation in immune mediated colitis by promoting T reg and increasing serum Il-10 levels [25]. So, the right microbioma equipment in the gut may help the mother to attain a strong contribution to the immune tolerance against the foetus.

\section{Following in pregnancy's immune tolerance's footsteps may cast light on future trends in human tissue and/or organ transplantation}

The induction of peripheral tolerance walks across the following steps: 1) the slow and increasing exposition of donor-derived soluble antigens to the recipient's immune system, like HLA-G does during pregnancy; 2) the down regulation of class I and II hystocompatibility antigen on organ to be transplanted; 3 ) sex hormones as progesteron has shown to be tolerogenic and it may be helpful in woman candidates to transplantation; 4) the check of nutritional status and the full supplementation of lacking nutritional factors; 5) the check of coagulation activation indexes to quickly diagnose and treat a thrombophylic state; 6) the check of immunoglobulin subclasses defect to quickly restore the idiotye-anti-idiotype network; 7) the check of a right gut microbioma.

As future approach, it's possible to imagine the following scenario. The slow and increasing exposition of donor-derived soluble antigens to the recipient's immune system may be attained by the prophylactic infusion of donor's plasma in recipient just before organ and/or tissue transplantation to allow the exposition of soluble donor-specific HLA antigens to the recipient's immune system in the aim to increase specific tolerance in the recipient. The down regulation of classic class I and II hystocompatibility antigens on donor's organ may be attained by the pretreatment of donor by immunosuppressive drugs as a steroid bolus just $1 \mathrm{~h}$ before organ collection (for example methylprednisolone $1 \mathrm{mg}$ / $\mathrm{kg} \mathrm{ev.).} \mathrm{It's} \mathrm{possible} \mathrm{to} \mathrm{think} \mathrm{about} \mathrm{the} \mathrm{therapeutic} \mathrm{use} \mathrm{of} \mathrm{hormones} \mathrm{as}$ tolerogenic factors: for example, the administration of progesterone in women candidates to transplantation before and after the transplant should increase the chances to reach immune tolerance against transplant, like as successful immune tolerance during pregnancy (a tentative period of six months should be suitable). Moreover, checking nutritional status after tissue and/or organ transplantation and after all procedures jeopardizing cell proliferation after surgery and/or chemical or radiobiological treatment, is pivotal to ensure the right cell proliferation thanks to administration of lacking nutritional factors as VitaminB12, Vitamin D, folic acid and iron, so ensuring the right DNA synthesis of normal proliferating cells in the transplant. That's mandatory when the repopulation of normal cells ( blood and/or organ cells) is entrusted to clonal proliferation of few cells as stem cells after bone marrow transplantation or vascular and cardiac cells after cardiosurgery. The check of coagulation activation indexes as d-dimer test are always mandatory both before and after transplantation to quickly diagnose and treat thrombotic state which may jeopardize the right blood flow to the transplanted organ. The check of immunoglobulin subclasses may help to diagnose immunoglobulin subclasses deficiency (particularly IgG1 and IgG3) and to treat it. In the case of exacting surgical interventions, as heart or pulmonary surgery, it should be advisable to include in the therapeutic arsenal the iv. infusion of whole immunoglobulins the day after transplantation $(200 \mathrm{mg} / \mathrm{kg})$, immune modulating agents aimed both to lower the vulnerability to infections and to increase the transplant recipients immune tolerance thanks to the restoration of idiotype-antiidiotype network. As to gut microbioma, it's very important to check the bowel function in patient candidates to transplant. If necessary, after eradicating the resident microbioma with antibiotics, it should be advisable to administrate proper probiotics aimed to elicitate immune tolerance of the transplant recipient, as Bifidobacteria and Lactobacilli, 1 month before the transplant. When possible it should be advisable to allow the tolerogenic exposition of the antigen to the oral mucosa of the recipient; for example, in the case of xenotransplant of cardiac porcine valves in humans, the prolonged oral exposition of porcine meat during mastication ( $3 \mathrm{~min}$.), may help to reach immune tolerance against xenotransplant in patients sensitized to suine proteins. Nothing prohibits to join these treatments to the others put in play during tissue and/or organ transplantation.

Anyway, the best aim in organ and tissue transplantation is to do all possible things to help the patients to live as better and longer as possible with a new organ's long-lasting function; the knowledge of the multiple mechanisms put in play by the women during pregnancy may help to attain this aim.

\section{Funding and Conflicts of Interest}

Author certify the absence of economic or other types of conflicts of interests regarding the present review.

\section{References}

1. Davies CJ (2007) Why is the fetal allograft not rejected? J Anim Sci 85: E32-E35. [Crossref]

2. LeMaoult J, Krawice-Radanne I, Dausset J, Carosella ED (2004) HLA-G1-expressing antigen-presenting cells induce immunosuppressive CD4+ T cells. Proc Natl Acad Sci US A 101: 7064-7069. [Crossref]

3. Hunt JS, Petroff MG, McIntire RH, Ober C (2005) HLAG and immune tolerance in pregnancy. FASEB J 19: 681-693. [Crossref]

4. Ishitani A, Sageshima N, Lee N, Dorofeeva N, Hatake K, Marquardt H, et al. (2003) Protein expression andpeptide binding suggest unique and interacting functional roles for HLA-E, F, and G in maternal-placental immune recognition. J Immunol 171: 13761384.

5. Kirana C, Ruszkiewicz A, Stubbs RS, Hardingham JE, Hewett PJ, et al. (2017) Soluble HLA-G is a differential prognostic marker in sequential colorectal cancer disease stages. Int J Cancer 140: 2577-2586. [Crossref]

6. Wang X, Sherman A, Liao G, Leong KW, Daniell H, et al. (2013) Mechanism of Oral Tolerance Induction to Therapeutic Proteins. Adv Drug Deliv Rev 65: 759-773. [Crossref]

7. Baek JO, Roh J, Jung Y (2017) Oral tolerance inhibits atopic dermatitis-like type 2 inflammation in mice by modulating immune microenvironments. Allergy 72: 397-406. [Crossref]

8. Faria AM, Weiner HL (2006) Oral tolerance: Therapeutic implications for autoimmune diseases. Clin Dev Immunol 13: 143-157. [Crossref]

9. Chinthrajah RS, Hernandez JD, Boyd SD, Galli SJ, Nadeau KC (2016) Molecular and cellular mechanisms of food allergy and food tolerance. J Allergy Clin Immunol 137: 984-997. [Crossref]

10. Lissauer D, Eldershaw SA, Inman CF, Coomarasamy A, Moss PA, et al. (2015) Progesterone promotes maternal-fetal tolerance by reducing human maternal T-cell polyfunctionality and inducing a specific cytokine profile. Eur J Immunol 45: 28582872. [Crossref]

11. Szekeres-Bartho J, Polgar B, Kozma N, Miko E, Par G, Szereday L, et al. (2005) Progesterone-dependent immunomodulation. Chem Immunol Allergy 89: 118-125. [Crossref] 
12. Trigunaite A, Dimo J, Jørgensen TN (2015) Suppressive effects of androgens on the immune system. Cell Immunol 294: 87-94. [Crossref]

13. Elkin M, Cohen I, Zcharia E, Orgel A, Guata-Rangini Z, et al. (2003) Regulation of heparanase gene expression by estrogen in breast cancer. Cancer Res 63: 8821-8826.

14. US Preventive Services Task Force, Bibbins-Domingo K, Grossman DC, Curry SJ, Davidson KW, et al. (2017) Folic Acid Supplementation for the Prevention of Neural Tube Defects: US Preventive Services Task Force Recommendation Statement. JAMA 317: 183-189. [Crossref]

15. Stanhewicz AE, Kenney WL (2017) Role of folic acid in nitric oxide bioavailability and vascular endothelial function. Nutr Rev 75: 61-70. [Crossref]

16. Xu J, Jia X, Gu Y, Lewis DF, Gu X, et al. (2017) Vitamin D reduces oxidative stressinduced procaspase-3/ROCK1 activation and MP release by placental trophoblasts. $J$ Clin Endocrinol Metab 102: 2100-2110. [Crossref]

17. Zhang HH, Chen JC, Sheibani L, Lechuga TJ, Chen DB (2017) Pregnancy augments VEGF-stimulated in vitro angiogenesis and vasodilator (NO and $\mathrm{H} 2 \mathrm{~S}$ ) production in human uterine artery endothelial cells. J Clin Endocrinol Metab.

18. Adu-Bonsaffoh K, Antwi DA, Gyan B, Obed SA (2017) Endothelial dysfunction in the pathogenesis of pre-eclampsia in Ghanaian women. BMC Physiol 17: 5. [Crossref]

19. Chapin J, Terry HS, Kleinert D, Laurence J (2016) The role of complement activation in thrombosis and hemolytic anemias. Transfus Apher Sci 54: 191-198. [Crossref]

20. Dusse LM, Alpoim PN, Lwaleed BA, de Sousa LP, Carvalho MD, et al. (2013) Is there a link between endothelial dysfunction, coagulation activation and nitric oxide synthesis in preeclampsia? Clin Chim Acta 415: 226-229.

21. Lavasani S, Dzhambazov B, Nouri M, Fåk F, Buske S, et al. (2010) A Novel Probiotic Mixture Exerts a Therapeutic Effect on Experimental Autoimmune Encephalomyelitis Mediated by IL-10 Producing Regulatory T Cells PLoS One 5: 1-11.
22. Round JL, Mazmanian SK (2010) Inducible Foxp3 ${ }^{+}$regulatory T-cell development by a commensal bacterium of the intestinal microbiota. Proc Natl Acad Sci U S A 107: 12204-12209.

23. Berni Canani R, Sangwan N, Stefka AT, Nocerino R, Paparo L, et al. (2016) Lactobacillus rhamnosus GG supplemented formula expands butyrate-producing bacterial strains in food allergic infants. ISME J 100: 742-750. [Crossref]

24. Furrie E, Macfarlane S, Thomson G, Macfarlane GT (2005) Toll-like receptors-2, -3 and -4 expression patterns on human colon and their regulation by mucosal-associated bacteria. Immunology 115: 565-574.

25. Ya'acov AB, Lichtenstein Y, Zolotarov L, Ilan Y (2015) The gut microbiome as a target for regulatory $\mathrm{T}$ cell-based immunotherapy: induction of regulatory lymphocytes by oral administration of anti-LPS enriched colostrum alleviates immune mediated colitis. BMC Gastroenterol 154: 1-8. [Crossref]

26. Habicht A, Dada S, Jurewicz M, Fife BT, Yagita H, et al. (2007) A Link between PDL1 and T Regulatory Cells in Fetomaternal Tolerance. J Immunol 179: 5211-5219.

27. Schumacher A, Brachwitz N, Sohr S, Engeland K, Langwisch S, et al. (2009) Human Chorionic Gonadotropin Attracts Regulatory T Cells into the Fetal-Maternal Interface during Early Human Pregnancy. J Immunol 182: 5488-5497.

28. Saito S, Shiozaki A, Nakashima A, Sakai M, Sasaki Y (2007) The role of the immune system in preeclampsia. Mol Aspects Med 28: 192-209.

29. Manfredi G, Dell'Aera L, Liguori R (2015) Overcoming recurrent spontaneous abortions in women suffering from IgG subclass deficiency: high efficiency of low dose intravenous immunoglobulins treatment. Eur Ann Allergy Clin Immunol 47: 91-94.

30. Stoheger ZM, Dayan M, Tcheriack A, Green L, Toledo S, et al. (2003) Modulation of autoreactive responses of peripheral blood lymphocytes of patients with systemic lupus erythematosus by peptides based on human and murine anti-DNA autoantibodies. Clin Exp Immunol 131: 385-392.

Copyright: (C2018 Manfredi G. This is an open-access article distributed under the terms of the Creative Commons Attribution License, which permits unrestricted use, distribution, and reproduction in any medium, provided the original author and source are credited. 